\title{
Electrochemical Synthesis and Electro-Optical Properties of Dibenzothiophene/Thiophene Conjugated Polymers With Stepwise Enhanced Conjugation Lengths
}

OPEN ACCESS

Edited by:

Haichang Zhang,

Qingdao University of Science and

Technology, China

Reviewed by:

Kun Yang,

Southern University of Science and

Technology, China

Maning Liu,

Tampere University, Finland

${ }^{*}$ Correspondence:

Kaiwen Lin

kevinlin1990@163.com

Xi LiU

liuxi_wyu@163.com

Specialty section:

This article was submitted to

Organic Chemistry,

a section of the journal

Frontiers in Chemistry

Received: 07 July 2020

Accepted: 04 August 2020

Published: 08 September 2020

Citation:

Lin K, Li C, Tao W, Huang J, Wu Q,

Liu Z, Zhang Y, Wang $D$ and Liu $X$ (2020) Electrochemical Synthesis and

Electro-Optical Properties of

Dibenzothiophene/Thiophene

Conjugated Polymers With Stepwise

Enhanced Conjugation Lengths.

Front. Chem. 8:819.

doi: 10.3389/fchem.2020.00819

\section{Kaiwen Lin ${ }^{1 *}$, Caiting $\mathrm{Li}^{2}$, Wang $\mathrm{TaO}^{3}$, Jilong Huang ${ }^{1}$, Qinghua $\mathrm{Wu}^{2}$, Zijin $\mathrm{Liu}^{2}$, Yangfan Zhang ${ }^{2}$, Da Wang ${ }^{3}$ and Xi Liu ${ }^{2 *}$}

${ }^{1}$ Department of Materials and Food, Zhongshan Institute, University of Electronic Science and Technology of China, Zhongshan, China, ${ }^{2}$ School of Textile Materials and Engineering, Wuyi University, Jiangmen, China, ${ }^{3}$ School of Applied Physics and Materials, Wuyi University, Jiangmen, China

A total of six conjugated polymers, namely PDBT-Th, PDBT-Th:Th, PDBT-2Th, PDBTTh:2Th, PDBT-2Th:Th, and PDBT-2Th:2Th, consisting of dibenzothiophene, thiophene, and bithiophene were electrochemically synthesized. Their electrochemical and electrochromic properties were investigated in relation to the conjugation chain lengths of the thiophene units in the conjugated backbones. Density functional theory (DFT) calculations showed that longer conjugation lengths resulted in decreased $\mathrm{HOMO}-\mathrm{LUMO}$ gaps in the polymers. The optical band gaps $\left(E_{\mathrm{g}, \mathrm{opt}}\right)$ and electrochemical band gaps $\left(E_{\mathrm{g}, \mathrm{cv}}\right)$ were decreased from PDBT-Th to PDBT-Th:Th, however, PDBT-Th:2Th, PDBT-2Th, PDBT-2Th:Th and PDBT-2Th:2Th displayed the similar band gaps. The conjugation length increments significantly improved the electrochemical stability of the conjugated polymers and exhibited reversible color changes due to the formation of polarons and bipolarons. The results suggest that the conjugated polymers prepared herein are promising candidates for fabricating flexible organic electrochromic devices.

Keywords: dibenzothiophene, electrochemical synthesis, electrochromism, conjugation lengths, color changes, electrochemical stability

\section{INTRODUCTION}

It is indispensable for the preparation of high-performance conjugated polymers and development of state-of-the-art applications to explore the structure-performance relationship of conjugated polymers. Breakthroughs in organic optoelectronics, including organic solar cells, dye-sensitized solar cells, organic field effect transistors, and electrochromism (Zhang et al., 2013; Jin et al., 2014; Zhou et al., 2015; Li et al., 2019a), has been performed by altering the main conjugation length. Strategies for altering the main conjugation length of organic optoelectronic materials include chemical and electrochemical polymerization methods (Jin et al., 2014; Zhou et al., 2015).

Electrochemical polymerization is often used in organic optoelectronics and employs a working electrode on which the polymer films simultaneous polymerize and are deposited by an applied voltage (Li et al., 2009; Jiang et al., 2018). Electrochemical polymer preparation exhibits several advantages: (1) It is high-throughput for synthesizing polymer films, formed only on the electrodes without any observed in solution; (2) It is highly efficient for synthesizing polymer films, 
since the reaction can be conducted in several seconds or minutes, while conventional chemical synthesis of a similar polymer requires several hours or days; (3) The electropolymerization method uses cheap supporting electrolytes instead of specific catalysts and expensive complexants for solution-phase synthesis; (4) The reaction can be performed at room temperature, whereas conventional chemical synthesis usually requires harsh conditions with high temperatures under an inert atmosphere (Gu et al., 2015; Yuan and Lei, 2020). The extension of the main chain conjugation length via electrochemical polymerization is effectively concentrated in electrochromism (Ak et al., 2008; Camurlu et al., 2008; Kavak et al., 2015; Sheberla et al., 2015; Gu et al., 2018; Li et al., 2019b; Zhang et al., 2019; Lu et al., 2020). Electrochromism is often defined as the visible and reversible changes in the transmittance and color of a material caused by an applied voltage (Argun et al., 2004; Beaujuge and Reynolds, 2010; Lin et al., 2017). For example, Zhao et al., verified the stepwise enhancement of the electrochemical and electrochromic performances of polyselenophene via electropolymerization of mono-, bi-, and triselenophene. Polyselenophene that was electropolymerized from triselenophene exhibited the lowest optical band gap $(1.72 \mathrm{eV})$, highest redox stability, as well as the best electrochromic nature of optical contrast up to $75 \%$, coloration efficiency up to $450 \mathrm{~cm}^{2} \mathrm{C}^{-1}$, and switching time $(0.7$ and $0.4 \mathrm{~s}$ for oxidation and reduction, respectively) compared to polyselenophene prepared from mono- and biselenophene ( $\mathrm{Lu}$ et al., 2020). Zhang et al., prepared a cross-linked copolymer (pTPhSNS-EDOT) via electrochemical polymerization that exhibited a fast coloring time of $0.58 \mathrm{~s}$ and discoloring time of
$0.38 \mathrm{~s}$, high optical contrast of $40 \%$, excellent color stability, and improved color memory behavior compared to the pTPhSNS homopolymer (Dai et al., 2017). Therefore, extension of the main chain conjugation length is beneficial for obtaining excellent electrochemical and electrochromic properties.

Herein, thiophene, and thiophene derivatives were used to construct electrochromic polymers with stepwise enhancement of the main chain conjugation lengths via electrochemical copolymerization. The relationship between the main chain conjugation length and electrochromic properties, as well as the electrochemical redox activity and stability of the conjugated polymer, were studied in detail. Significantly, this study provides theoretical guidance for the development of related fields.

\section{RESULT AND DISCUSSION \\ Synthesis and Characterization}

The synthetic routes for the monomers (DBT-Th and DBT-2Th) and electrochemical polymers (PDBT-Th, PDBT-2Th, PDBTTh:Th, PDBT-Th:2Th, PDBT-2Th:Th, and PDBT-2Th:2Th) are illustrated in Scheme 1. Poly[2,8-bis-(thiophen-2-yl)dibenzothiophene] (PDBT-Th) and poly[2,8-Bis-(bithiophen2-yl)-dibenzothiophene] (PDBT-2Th) were prepared from the 2,8-bis-(thiophen-2-yl)-dibenzothiophene (DBT-Th) and 2,8-Bis-(bithiophen-2-yl)-dibenzothiophene (DBT-2Th) monomers $\left(0.01 \mathrm{~mol} \mathrm{~L}^{-1}\right)$ and $\mathrm{Bu}_{4} \mathrm{NPF}_{6}\left(0.1 \mathrm{~mol} \mathrm{~L}^{-1}\right)$ in DCM via electrochemical polymerization. PDBT-Th:Th and PDBT-Th:2Th were electrochemical polymerized from $0.005 \mathrm{~mol}$ $\mathrm{L}^{-1}$ DBT-Th and $0.005 \mathrm{~mol} \mathrm{~L}-1$ thiophene $(\mathrm{Th}) /$ bithiophene (2Th), respectively. PDBT-2Th:Th and PDBT-2Th:2Th were

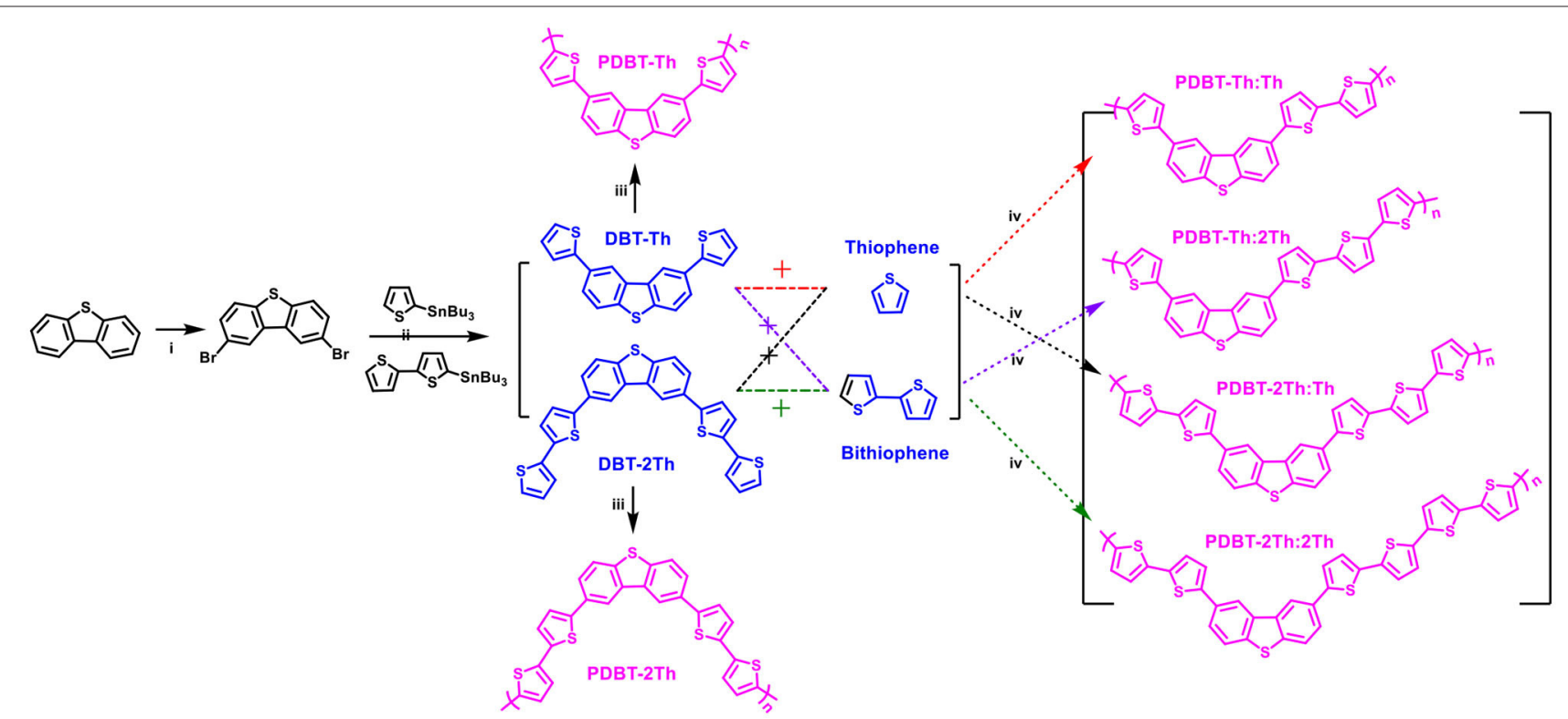

SCHEME 1 | Synthetic routes of monomers (DBT-Th and DBT-2Th) and electrochemical polymers (PDBT-Th, PDBT-2Th, PDBT-Th:Th, PDBT-Th:2Th, PDBT-2Th:Th, and PDBT-2Th:2Th). Reagents and conditions: (i) $\mathrm{CHCl}_{3}, \mathrm{Br}_{2}$ (2.2 eq.), $\mathrm{N}_{2}$ (ii) $\mathrm{Pd}\left(\mathrm{PPh}_{3}\right)_{4}$, chlorobenzene, $90^{\circ} \mathrm{C}$, (iii) electrochemical polymerization, $\mathrm{CH}_{2} \mathrm{Cl}_{2}-\mathrm{Bu}_{4} \mathrm{NPF}_{6}$, (iv) electrochemical copolymerization, $\mathrm{CH}_{2} \mathrm{Cl}_{2}-\mathrm{Bu}_{4} \mathrm{NPF}_{6}$. 
obtained from $0.005 \mathrm{~mol} \mathrm{~L}^{-1}$ DBT-2Th and $0.005 \mathrm{~mol} \mathrm{~L}{ }^{-1}$ $\mathrm{Th} / 2 \mathrm{Th}$ via electrochemical copolymerization. From the synthetic routes of DBT-Th and DBT-2Th, dibenzothiophene (DBT) was used to prepare the corresponding bromide (2,8-dibromodibenzothiophene) via bromination. The above dibromide was reacted with tributyltin substituted $\mathrm{Th} / 2 \mathrm{Th}$ to afford the target products using a $\mathrm{Pd}\left(\mathrm{PPh}_{3}\right)_{4}$ catalyst. The ${ }^{1} \mathrm{H}$ NMR and ${ }^{13} \mathrm{H}$ NMR spectra of target monomers are presented in Figures S1-S4.

\section{Electrochemical Polymerization of DBT-Th, DBT-2Th and Electrochemical Copolymerization of DBT-Th, DBT-2Th, Th, and $2 \mathrm{Th}$}

From their anodic oxidation curves (Figure S5), the onset oxidation potentials $\left(E_{\text {onset }}\right)$ were initiated at 1.11, 1.30, 0.85, 1.27, 1.07 , and $0.98 \mathrm{~V}$ for DBT-Th, DBT-Th:Th, DBT-2Th, DBT-Th:2Th, DBT-2Th:Th, and DBT-2Th:2Th, respectively. The polymers were prepared by the potentiostatic method (adding about $0.2 \mathrm{~V}$ of $E_{\text {onset }}$ ) along with similar charge of about $5 \mathrm{mC}$. In this electrochemical deposition condition, the thickness of polymer films were about $200 \mathrm{~nm}$, which is in agreement with the reported research (Lin et al., 2020b).

Figure 1 shows the obtained cyclic voltammograms (CVs) corresponding to the potentiodynamic electropolymerization of the polymeric precursor monomers DBT-Th and DBT-2Th as well as the potentiodynamic electrochemical copolymerization of the polymeric precursor comonomers DBT-Th, DBT-2Th, Th, and 2Th. The current density increased gradually as a function of scanning cycles for all systems. Meanwhile, the corresponding conducting polymers on the working electrode grew prominently, indicating that the as-prepared conducting polymers exhibited good electrochemical redox activity. Meanwhile, all systems exhibited an increasingly obvious voltage drop $(\Delta \mathrm{V})$ from 0.6 to $1.0 \mathrm{~V}$ of the reduction peak with stepwise enhanced conjugation lengths, which were ascribed to the wide distribution of polymer chain lengths. Because of the additional potential required to balance the increased polymeric electrical resistance and slow mass transport, an obvious potential shift of the anodic and cathodic peaks was observed during polymer growth (Chen and Xue, 2005; Lin et al., 2020a; Lu et al., 2020).

\section{Theoretical Calculations}

The ground-state optimized molecular geometries and frontier molecular orbital distributions of DBT-Th, DBT-Th:Th, DBT2Th, DBT-Th:2Th, DBT-2Th:Th, and DBT-2Th:2Th were determined using density functional theory (DFT) by Gaussian 09 at the B3LYP/6-31G(d,p) level (Figure 2). All optimized molecular geometries exhibited slightly twisted, n-shaped structures, with dihedral angles of $<31^{\circ}$ owing to the steric hindrance effect. The dihedral angles of DBT and adjacent thiophene decreased with enhanced conjugation lengths, which resulted in improved regularity. For all models, the electron density distribution of the lowest unoccupied molecular orbitals
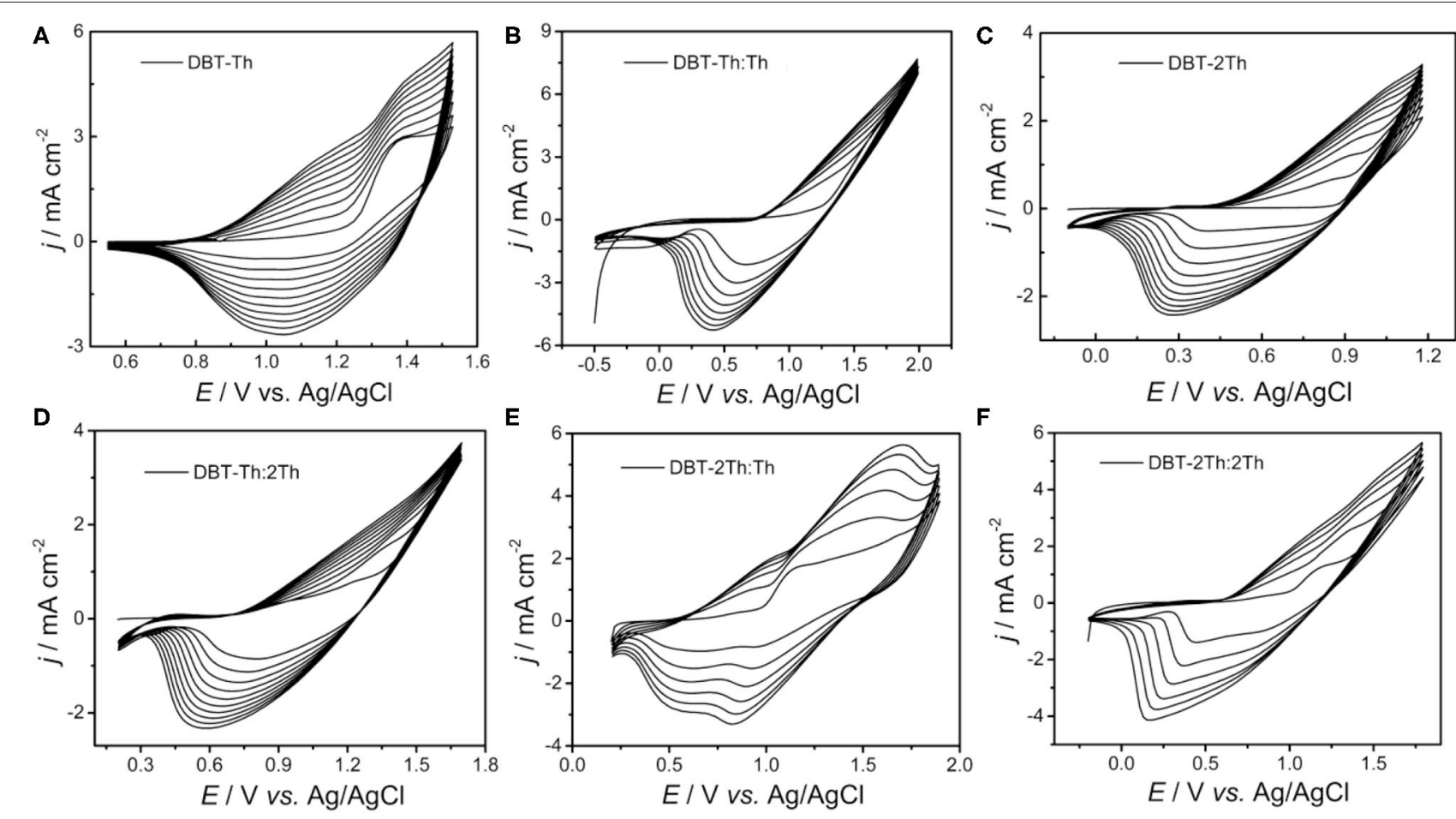

FIGURE 1 | Cyclic voltammograms of DBT-Th (A), DBT-Th: Th (B), DBT-2Th (C), DBT-Th: 2Th (D), DBT-2Th: Th (E), and DBT-2Th: 2Th (F) in CH ${ }_{2} \mathrm{Cl}_{2}-\mathrm{Bu}_{4} \mathrm{NPF}_{6}$. Potential scan rate: $100 \mathrm{mV} \mathrm{s}^{-1}$. 


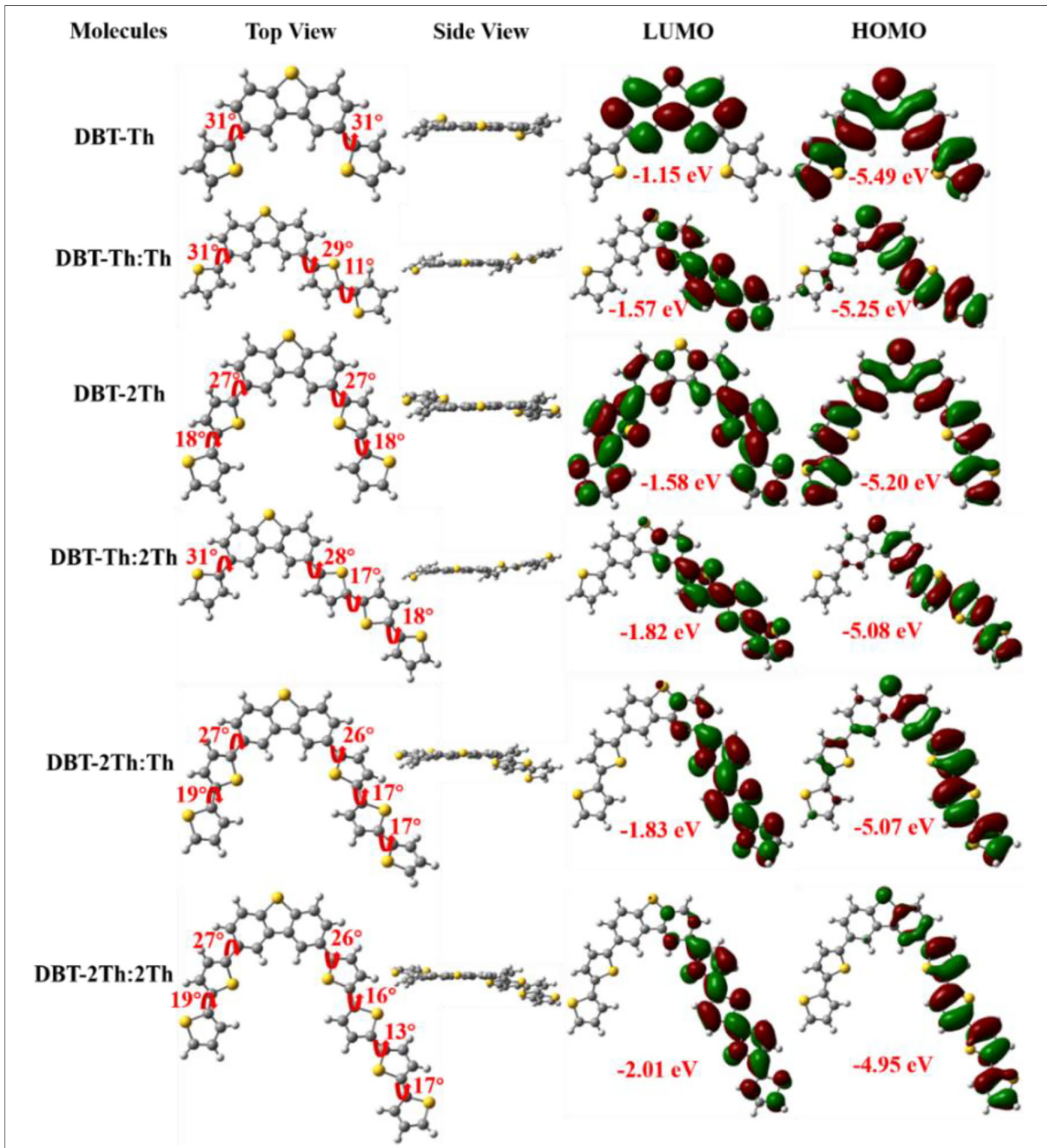

FIGURE 2 | Optimized molecular geometries and frontier molecular orbital distributions of DBT-Th, DBT-Th:Th, DBT-2Th, DBT-Th:2Th, DBT-2Th:Th, and DBT-2Th:2Th obtained from DFT by Gaussian 09 at the B3LYP/6-31G(d,p) level.

(LUMOs) and highest occupied molecular orbitals (HOMOs) were localized in the sectional conjugated skeleton. The longer the conjugation lengths resulted in lower energy LUMO and
HOMO levels (Liu et al., 2017). The HOMO-LUMO gaps of all models gradually decreased from 4.34 to $2.94 \mathrm{eV}$ with stepwise enhancement of the conjugation lengths. 

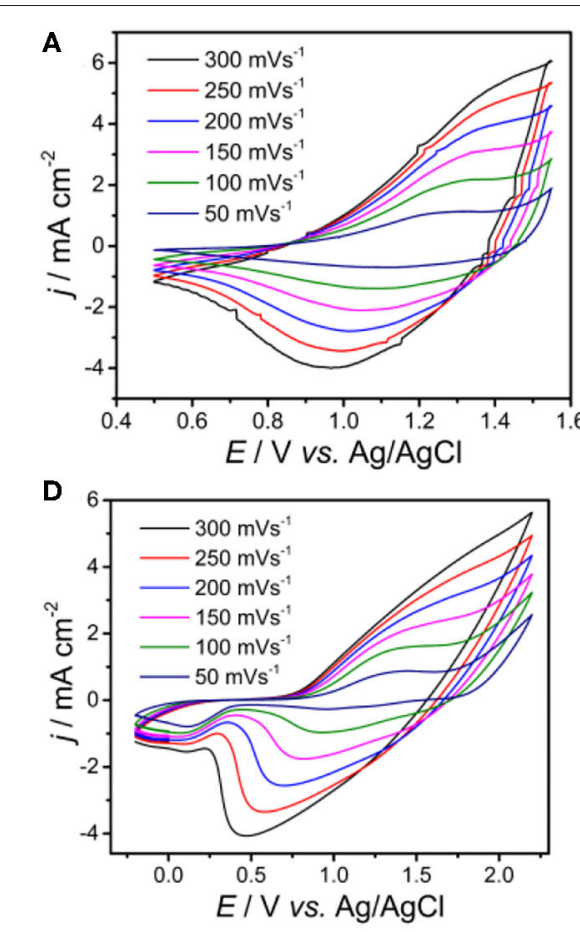
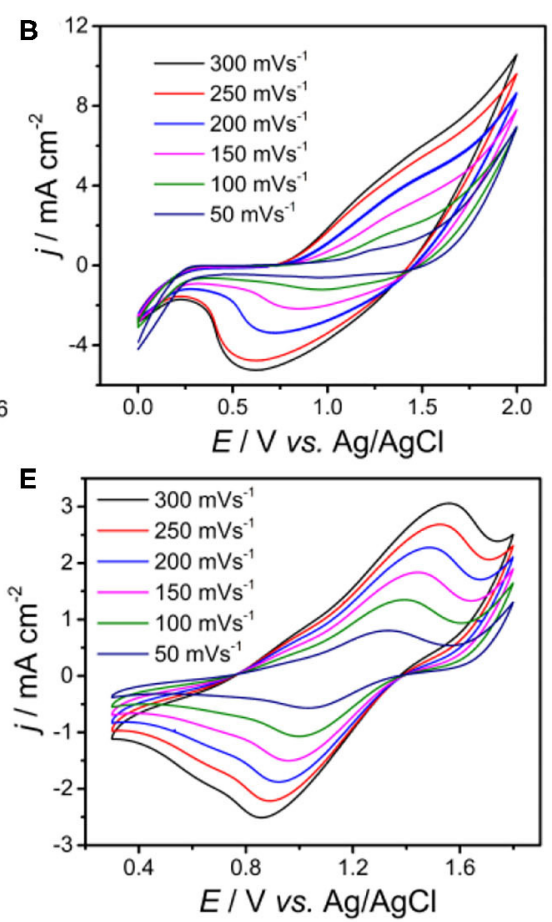

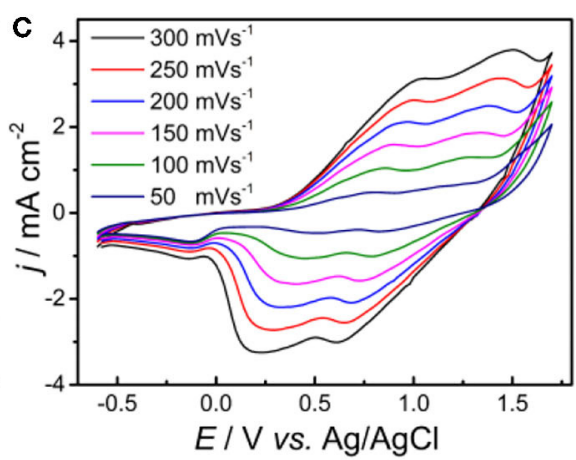

$\mathbf{F}$

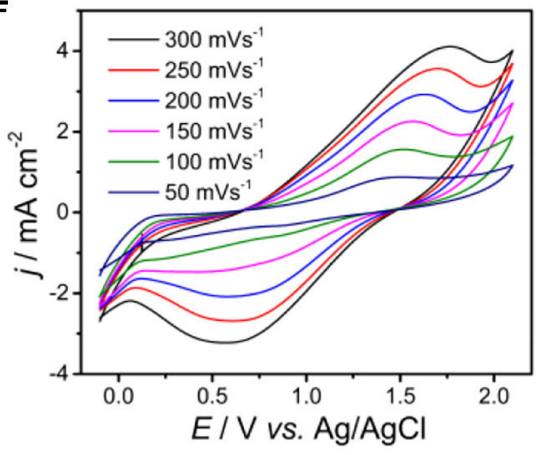

FIGURE 3 | Cyclic voltammograms of the electrochemical polymer PDBT-Th (A), PDBT-Th:Th (B), PDBT-2Th (C), PDBT-Th:2Th (D), PDBT-2Th:Th (E), and PDBT-2Th:2Th (F) modified Pt electrodes in monomer-free $\mathrm{CH}_{2} \mathrm{Cl}_{2}-\mathrm{Bu}_{4} \mathrm{NPF}_{6}\left(0.10 \mathrm{~mol} \mathrm{~L}^{-1}\right)$ at potential scan rates of 300, $250,200,150,100$, and 50 $\mathrm{mV} \mathrm{s}^{-1}$.

TABLE 1 | Electrochemical, optical properties and theoretical calculation of polymers.

\begin{tabular}{|c|c|c|c|c|c|c|c|c|c|}
\hline \multirow[t]{2}{*}{ Polymers } & \multirow[t]{2}{*}{$E_{\text {ox,onset }}(\mathbf{V})$} & \multirow[t]{2}{*}{$E_{\text {red,onset }}(\mathbf{V})$} & \multicolumn{2}{|c|}{ НОМо } & \multicolumn{2}{|c|}{ LUMO } & \multirow[t]{2}{*}{$E_{\mathrm{g}, \mathrm{cv}}(\mathrm{eV})$} & \multirow[t]{2}{*}{ HOMO-LUMO gaps (eV) } & \multirow[t]{2}{*}{$E_{\mathrm{g}, \mathrm{opt}}(\mathrm{eV})$} \\
\hline & & & $\begin{array}{l}\text { Experimental } \\
\text { value }\end{array}$ & $\begin{array}{c}\text { Theoretical } \\
\text { value }\end{array}$ & $\begin{array}{r}\text { Experim } \\
\text { value }\end{array}$ & $\begin{array}{l}\text { Theoretical } \\
\text { value }\end{array}$ & & & \\
\hline PDBT-Th & 0.88 & -1.95 & -5.68 & -5.10 & -2.85 & -1.73 & 2.83 & 3.37 & 2.59 \\
\hline PDBT-Th:Th & 0.70 & -1.75 & -5.50 & -5.00 & -3.05 & -1.97 & 2.45 & 3.03 & 2.53 \\
\hline PDBT-2Th & 0.55 & -1.74 & -5.35 & -4.90 & -3.06 & -2.09 & 2.29 & 2.81 & 2.25 \\
\hline PDBT-Th:2Th & 0.56 & -1.72 & -5.36 & -4.92 & -3.08 & -2.10 & 2.28 & 2.82 & 2.29 \\
\hline PDBT-2Th:Th & 0.54 & -1.75 & 5.34 & -4.84 & -3.05 & -2.19 & 2.29 & 2.65 & 2.24 \\
\hline PDBT-2Th:2Th & 0.52 & -1.77 & -5.32 & -4.81 & -3.03 & -2.25 & 2.29 & 2.56 & 2.23 \\
\hline
\end{tabular}

\section{Electrochemistry of the Polymers}

To obtain deeper insight of the electrochemical activity, the electrochemical behaviors of PDBT-Th, PDBT-Th:Th, PDBT-2Th, PDBT-Th:2Th, PDBT-2Th:Th, and PDBT-2Th:2Th were investigated via CVs in monomer-free $\mathrm{CH}_{2} \mathrm{Cl}_{2}-\mathrm{Bu}_{4} \mathrm{NPF}_{6}$ $\left(0.1 \mathrm{~mol} \mathrm{~L}^{-1}\right.$; Figure 3). All polymers were prepared using the potentiostatic method at a constant potential of $1.30 \mathrm{~V}$ for DBTTh, $1.50 \mathrm{~V}$ for DBT-Th:Th, $1.05 \mathrm{~V}$ for DBT-2Th, $1.45 \mathrm{~V}$ for DBTTh:2Th, 1.3 V for DBT-2Th:Th, and 1.2 V for DBT-2Th:2Th. All polymers showed obvious redox peaks with hysteresis (potential drift) between the anodic and cathodic peak potentials. The potential shifts of the redox peaks among the CVs were attributed to slow heterogeneous electron transfer, local rearrangement of the polymer chains, slow mutual transformation of various electronic species, and electronic charging of the interfacial exchange at the metal/polymer and polymer/solution interfaces (Inzelt et al., 2000).

In addition, the cyclic voltammetry was employed to evaluate the experimental HOMO and LUMO energy levels of polymers through the empirical Equations (1) and (2) in the Supporting Information (Sun et al., 2011). Meanwhile, the theoretical calculated HOMO/LUMO energy levels of polymers (simplified by two repeating units) were illustrated comparatively. The values were presented in Table 1. The experimental and theoretical calculated HOMO both exhibited up lifted values when the conjugation length was increased. The HOMO-LUMO gaps by theoretical calculation decreased gradually. The optical band gaps $\left(E_{\mathrm{g}, \mathrm{opt}}\right)$ and electrochemical band gaps $\left(E_{\mathrm{g}, \mathrm{cv}}\right)$ were decreased from PDBTTh to PDBT-Th:Th, however, PDBT-Th:2Th, PDBT-2Th, 

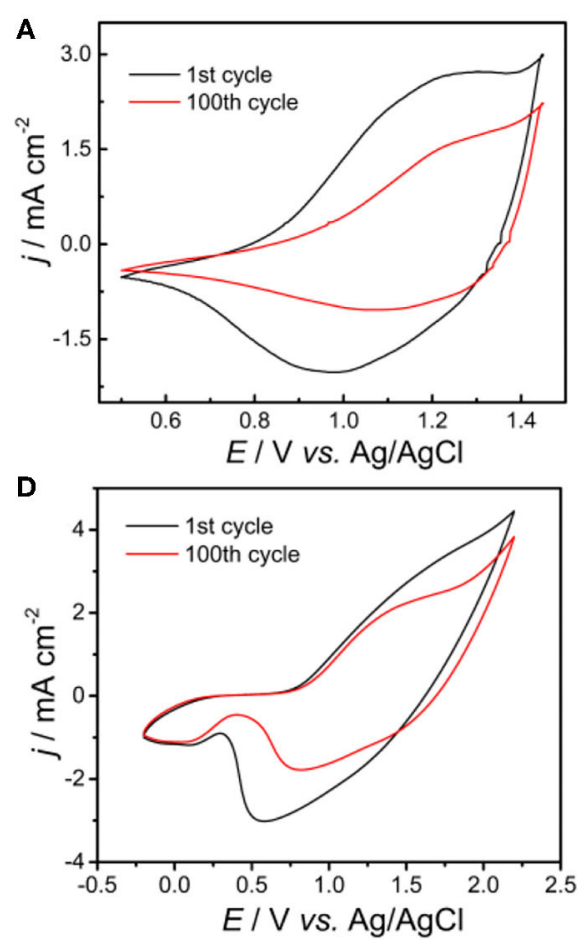
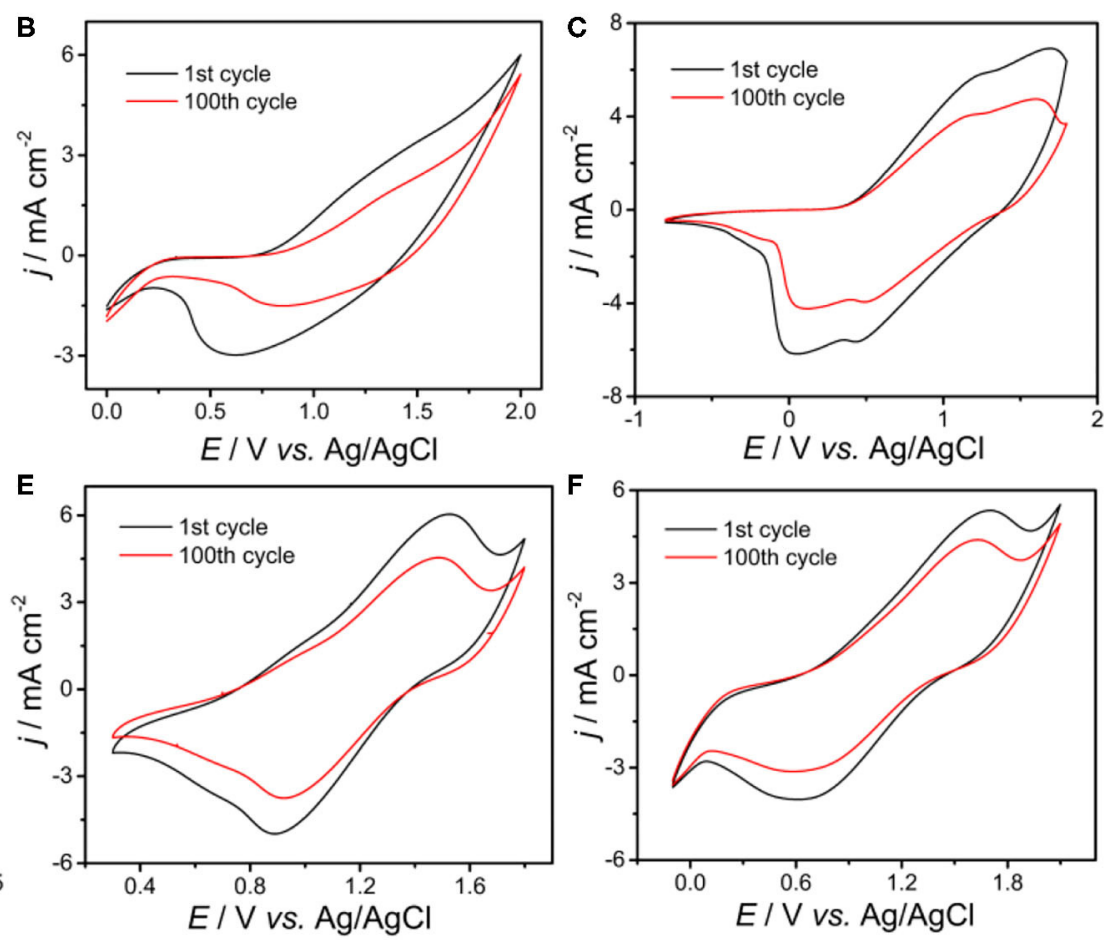

FIGURE 4 | CVs of PDBT-Th (A), PDBT-Th:Th (B), PDBT-2Th (C), PDBT-Th:2Th (D), PDBT-2Th:Th (E), and PDBT-2Th:2Th (F) cycled 100 times at a potential scan rate of $200 \mathrm{mV} \mathrm{s}^{-1}$ in monomer-free $\mathrm{CH}_{2} \mathrm{Cl}_{2}-\mathrm{Bu}_{4} \mathrm{NPF}_{6}\left(0.10 \mathrm{~mol} \mathrm{~L}{ }^{-1}\right)$.

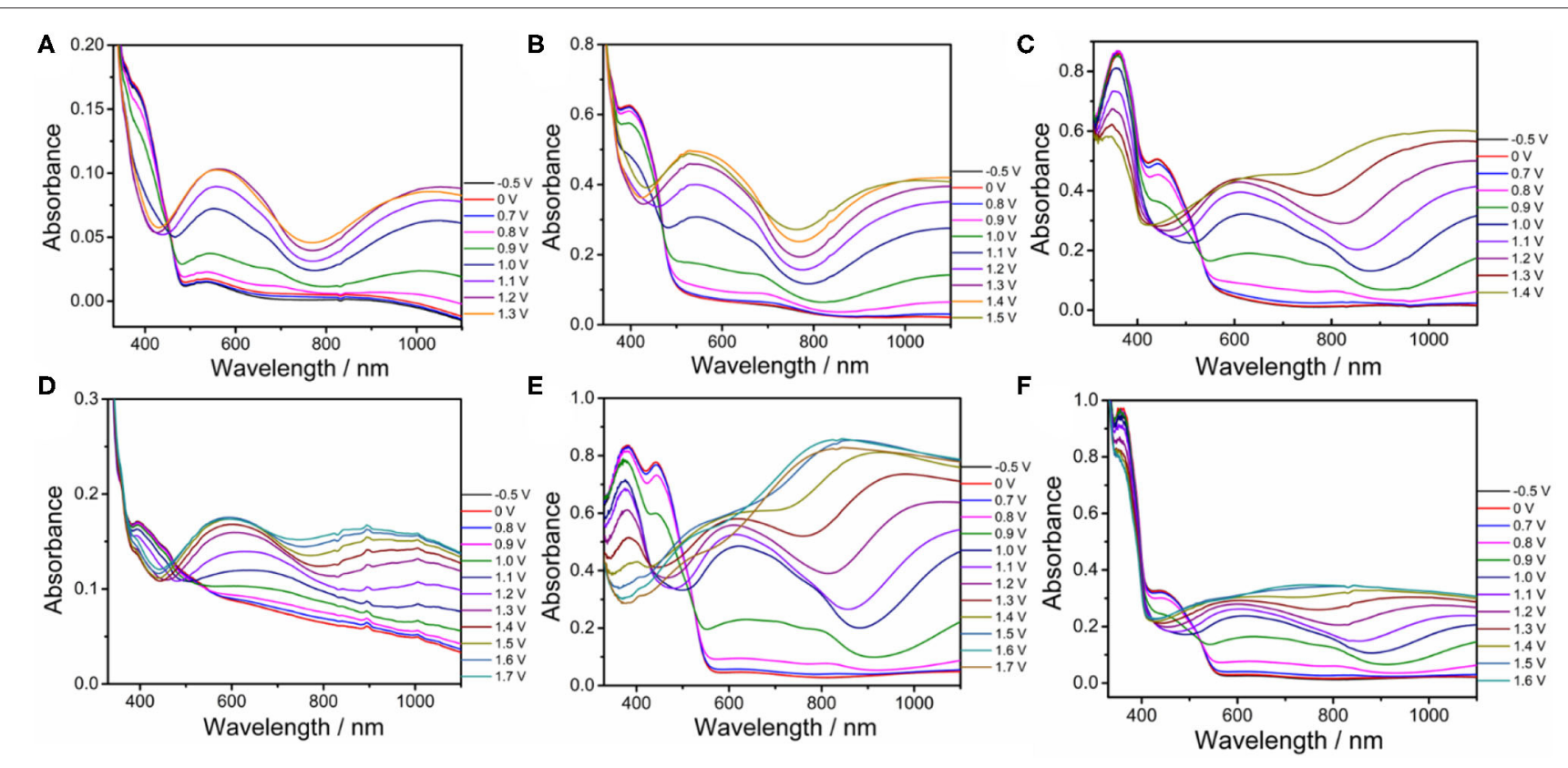

FIGURE 5 | Spectroelectrochemical traces for PDBT-Th (A), PDBT-Th:Th (B), PDBT-2Th (C), PDBT-Th:2Th (D), PDBT-2Th:Th (E), and PDBT-2Th:2Th (F) on ITO coated glass in $\mathrm{CH}_{3} \mathrm{CN}-\mathrm{Bu}_{4} \mathrm{NPF}_{6}\left(0.1 \mathrm{~mol} \mathrm{~L}{ }^{-1}\right)$. 
TABLE 2 | Colorimetric parameters for the prepared polymers.

\begin{tabular}{|c|c|c|c|c|c|}
\hline \multirow[t]{2}{*}{ Polymers } & \multicolumn{3}{|c|}{ CIE color coordinates } & \multicolumn{2}{|c|}{ Colors of polymers } \\
\hline & & Neutral & Oxidized & Neutral & Oxidized \\
\hline \multirow[t]{3}{*}{ PDBT-Th } & $L^{*}$ & 98.7235 & 92.2886 & & \\
\hline & $a^{*}$ & -2.2272 & 1.6750 & & \\
\hline & $b^{*}$ & 6.5666 & -3.7137 & & \\
\hline \multirow[t]{3}{*}{ PDBT-Th:Th } & $\mathrm{L}^{*}$ & 87.0708 & 67.1116 & & \\
\hline & $a^{*}$ & -8.4870 & 4.7474 & & \\
\hline & $b^{*}$ & 32.6390 & -3.4195 & & \\
\hline \multirow[t]{3}{*}{ PDBT-2Th } & $\mathrm{L}^{*}$ & 82.3156 & 79.7525 & & \\
\hline & $a^{*}$ & -1.3602 & -4.6198 & & \\
\hline & $b^{*}$ & 20.1580 & 4.5149 & & \\
\hline \multirow[t]{3}{*}{ PDBT-Th:2Th } & $L^{*}$ & 98.8370 & 94.9152 & & \\
\hline & $a^{*}$ & -0.2581 & -0.1172 & & \\
\hline & $b^{*}$ & 5.9035 & -4.5872 & & \\
\hline \multirow[t]{3}{*}{ PDBT-2Th:Th } & $\mathrm{L}^{*}$ & 84.1909 & 69.1127 & & \\
\hline & $a^{*}$ & 11.1086 & -2.1452 & & \\
\hline & $b^{*}$ & 63.0452 & -9.6235 & & \\
\hline \multirow[t]{3}{*}{ PDBT-2Th:2Th } & $L^{*}$ & 88.6797 & 76.6157 & & \\
\hline & $a^{*}$ & 6.0639 & -1.6808 & & \\
\hline & $b^{*}$ & 33.9582 & -4.6985 & & \\
\hline
\end{tabular}

PDBT-2Th:Th and PDBT-2Th:2Th displayed the similar band gaps.

The stabilities of all polymers were studied in a monomerfree $\mathrm{CH}_{2} \mathrm{Cl}_{2}-\mathrm{Bu}_{4} \mathrm{NPF}_{6}$ system. A total of 100 cycles were performed to study the doping and dedoping abilities of all polymers, as shown in Figure 4. The redox activity of PDBTTh was maintained at $56 \%$ after scanning 100 cycles, exhibiting generally good redox stability. However, increasing conjugation length significantly improved electrochemical stability, achieving moderate stability at $62,79,76,85$, and $90 \%$ remaining activity for PDBT-Th:Th, PDBT-2Th, PDBT-Th:2Th, PDBT-2Th:Th, and PDBT-2Th:2Th, respectively, after scanning 100 cycles. The improved electrochemical stability could benefit from the more stable thiophene bridge.

\section{Electrochromic Properties}

Spectroelectrochemical analyses were performed by recording the absorption changes of the polymers under different potentials (Figure 5). In the neutral state, all polymers showed the absorbance peaks centered at approximately $410 \mathrm{~nm}$ arising from the $\pi-\pi^{*}$ transition. DBT-2Th based polymers PDBT-2Th (c), PDBT-2Th:Th (e), and PDBT-2Th:2Th (f) clearly showed two absorbance peaks with red shift on the absorbance edges, which was ascribed to the enhanced conjugation lengths. The optical band gaps (Table 1, empirical Equation (3) in the Supporting Information) of the corresponding polymers were gradually reduced from $2.59 \mathrm{eV}$ (PDBT-Th) to $2.53 \mathrm{eV}$ (PDBT-Th:Th), $2.29 \mathrm{eV}$ (PDBT-Th:2Th), $2.25 \mathrm{eV}$ (PDBT-2Th), $2.24 \mathrm{eV}$ (PDBT2Th:Th), and $2.23 \mathrm{eV}$ (PDBT-2Th:2Th). With increasing effective conjugated chain length of those polymers by introducing thiophene as bridge unit, the optical band gaps were gradually reduced, and tend to the same. Notably, with increasing voltage, new bands resulting from the polaron at approximately $600 \mathrm{~nm}$ and bipolaron at approximately $1,000 \mathrm{~nm}$ increased in intensity (Zhu et al., 2009; Lu et al., 2014; Yang et al., 2014; Lin et al., 2015; Ming et al., 2020). During this process, all polymers displayed a conspicuous color change under doped (oxidized) and dedoped (neutral) conditions (Table 2). To determine the color change, the CIE $1976\left(\mathrm{~L}^{*}, \mathrm{a}^{*}, \mathrm{~b}^{*}\right)$ color space and photographs were determined, in which $L^{*}$ is the parameter of the lightness, $a^{*}$ is the red-green balance and $b^{*}$ is yellowblue balance $\left(-\mathrm{a}^{*}\right.$ and $+\mathrm{a}^{*}$ correspond to green and red and $-b^{*}$ and $+b^{*}$ correspond to blue and yellow, respectively) (Dyer et al., 2011). As presented in Table 2, PDBT-Th, PDBT-Th:Th, PDBT-2Th, and PDBT-Th:2Th showed $-\mathrm{a}^{*}$ and $+\mathrm{b}^{*}$ directions in the neutral state, therefore, the polymers exhibited yellow green color. PDBT-2Th:Th and PDBT-2Th:2Th exhibited noteworthy color of claybank (neutral state), which was attributed to the value of $+\mathrm{a}^{*}$ and $+\mathrm{b}^{*}$ directions. Isosbestic points at $450 \mathrm{~nm}$ of DBT-Th based polymers and $500 \mathrm{~nm}$ of DBT-2Th based polymers appeared in the spectra, indicating that these polymers were easily interconverted between neutral and oxidized states (Balan et al., 2011; Lin et al., 2020b).

\section{CONCLUSION}

Thiophene was used as a fundamental unit to progressively construct conjugated polymers of various lengths by electrochemical polymerization from polymeric precursor monomers (DBT-Th and DBT-2Th) and comonomers (thiophene and bithiophene). Theoretical DFT calculations, electrochemical, and electrochromic properties were measured and compared. All polymeric precursor systems exhibited increasingly significant voltage drops $(\Delta \mathrm{V})$ of their reduction peaks with enhanced conjugation length. All conjugated polymers exhibited decreased HOMO-LUMO gaps, significantly improved electrochemical stability, and noteworthy color changes when transitioning from the oxidized to neutral state with increasing conjugation length.

\section{DATA AVAILABILITY STATEMENT}

The raw data supporting the conclusions of this article will be made available by the authors, without undue reservation.

\section{AUTHOR CONTRIBUTIONS}

$\mathrm{KL}$ and $\mathrm{XL}$ designed the experiments. KL, JH, CL, WT, QW, ZL, and YZ performed the experiments. KL, DW, and XL analyzed the experimental results. KL and XL wrote the manuscript. All 
authors commented on the manuscript. KL and XL supervised the project.

\section{ACKNOWLEDGMENTS}

This work was supported by the Guangdong Basic and Applied Basic Research Fund (nos. 2019A1515110944 and 2019A1515010848) and the Youth Innovation Talent Project for the Universities of Guangdong Province (Grants: 2019KQNCX161 and 2019KQNCX190). We are also grateful to

\section{REFERENCES}

Ak, M., Tanyeli, C., Akhmedov, I. M., and Toppare, L. (2008). Optoelectrochemical Properties of the Copolymer of 2,5-Di(4-methylthiophen-2-yl)-1-(4nitrophenyl)-1H-pyrrole Monomer with 3,4-Ethylenedioxythiophene. Thin Solid Films 516, 4334-4341. doi: 10.1016/j.tsf.2008.01.013

Argun, A. A., Aubert, P. H., Thompson, B. C., Schwendeman, I., Gaupp, C. L., Hwang, J., et al. (2004). Chem. Mater. 16, 4401-4412. doi: 10.1021/cm049669l

Balan, A., Baran, D., and Toppare, L. (2011). Benzotriazole containing conjugated polymers for multipurpose organic electronic applications. Polym. Chem. 2, 1029-1043. doi: 10.1039/clpy00007a

Beaujuge, P. M., and Reynolds, J. R. (2010). Color control in $\pi$-conjugated organic polymers for use in electrochromic devices. Chem. Rev. 110, 268-320. doi: $10.1021 /$ cr900129a

Camurlu, P., Sahmetlioglu, E., Sahin, E., Akhmedov, I. M., Tanyeli, C., and Toppare, L. (2008). Fine tuning of color via copolymerization and its electrochromic device application. Thin Solid Films 516, 4139-4144. doi: 10.1016/j.tsf.2007.10.098

Chen, W., and Xue, G. (2005). Low potential electrochemical syntheses of heteroaromatic conducting polymers in a novel solvent system based on trifluroborate-ethyl ether. Prog. Polym. Sci. 30, 783-811. doi: 10.1016/j.progpolymsci.2005.03.002

Dai, Y. Y., Li, W. J., Qu, X. X., Liu, J., Yan, S. M., Ouyang, M., et al. (2017). Electrochemistry, electrochromic and color memory properties of polymer/copolymer based on novel dithienylpyrrole structure. Electrochim. Acta 229, 271-280. doi: 10.1016/j.electacta.2017.01.156

Dyer, A. L., Thompson, E. J., and Reynolds, J. R. (2011). Completing the color palette with spray-processable polymer electrochromics. ACS Appl. Mater. Interfaces 3, 1787-1795. doi: 10.1021/am200040p

Gu, C., Huang, N., Chen, Y. C., Qin, L. Q., Xu, H., Zhang, S. T., et al. (2015). $\pi$-conjugated microporous polymer films: designed synthesis, conducting properties, and photoenergy conversions. Angew. Chem. Int. Ed. 127, 13798-13802. doi: 10.1002/anie.201506570

Gu, H., Lin, K. W., Liu, X. M., Jian, N. N., Qu, K., and Xu, J. K. (2018). Enhanced redox stability and optical contrast of electrochromic copolymers from selenophene and 3-methylthiophene. J. Solid State Electrochem. 22, 599-611. doi: 10.1007/s10008-017-3803-0

Inzelt, G., Pineri, M., Schultze, J. W., and Vorotyntsev, M. A. (2000). Electron and proton conducting polymers: recent developments and prospects. Electrochim. Acta 45, 2403-2421. doi: 10.1016/S0013-4686(00)00329-7

Jiang, Y. Y., Xu, K., and Zeng, C. C. (2018). Use of electrochemistry in the synthesis of heterocyclic structures. Chem. Rev. 118, 4485-4540. doi: 10.1021/acs.chemrev.7b00271

Jin, X. H., Sheberla, D., Shimon, L. J. W., and Bendikov, M. (2014). Highly coplanar very long oligo(alkylfuran)s: a conjugated system with specific head-to-head defect. J. Am. Chem. Soc. 136, 2592-2601. doi: 10.1021/ja411842g

Kavak, E., Us, C. N., Yavuz, E., Kivrak, A., and Özkut, M. (2015). A camouflage material: p- and n-type dopable furan based low band gap electrochromic polymer and its EDOT based copolymer. Electrochim. Acta 182, 537-543. doi: 10.1016/j.electacta.2015.09.148

Li, C., Bai, H., and Shi, G. Q. (2009). Conducting polymer nanomaterials: electrosynthesis and applications. Chem. Soc. Rev. 38, 2397-2409. doi: $10.1039 / \mathrm{b} 816681 \mathrm{c}$
Zhongshan Science and Technology Public Projects (2019B2061) and PhD Early Development Program of University of Electronic Science and Technology of China Zhongshan Institute (419YKQN16) for their financial support of this study.

\section{SUPPLEMENTARY MATERIAL}

The Supplementary Material for this article can be found online at: https://www.frontiersin.org/articles/10.3389/fchem. 2020.00819/full\#supplementary-material

Li, C. T., Kuo, Y. L., Kumar, C. P., Huang, P. T., and Lin, J. T. (2019a). Tetraphenylethylene tethered phenothiazine based double-anchored sensitizers for high performance dye-sensitized solar cells. J. Mater. Chem. A 7, 23225-23233. doi: 10.1039/c9ta09025h

Li, W. J., Luo, F. F., Zhang, L., Yan, S. M., Zhao, R. Y., Ren, N., et al. (2019b). Synthesis, electrochemistry, and electrochromic properties of branched thiophene polymers with different conjugation lengths. J. Polym. Sci. Pol. Chem. 57, 2340. doi: 10.1002/pola.29538

Lin, K. W., Chen, S., Lu, B. Y., and Xu, J. K. (2017). Hybrid $\pi$-conjugated polymers from dibenzo pentacyclic centers: precursor design, electrosynthesis and electrochromics. Sci. China Chem. 60, 38-53. doi: 10.1007/s11426-0160298-2

Lin, K. W., Jian, N. N., Zhang, X. B., Zhang, Y. S., Ming, S. L., Zhen, S. J., et al. (2020b). Star-shaped trithiophene and hexathiophene functionalized truxenes: synthesis, electropolymerization, and electrochromism. React. Funct. Polym. 154:104674. doi: 10.1016/j.reactfunctpolym.2020. 104674

Lin, K. W., Ming, S. L., Chen, S., Zhang, X. B., Wang, K., Wang, Y. H., et al. (2020a). Yellow-to-transmissive Electrochromic Poly(dibenzothiophene/dibenzofuran-bithiophene). J. Solid State Electrochem. 24, 1387-1396. doi: 10.1007/s10008-020-04623-0

Lin, K. W., Ming, S. L., Zhen, S. J., Zhao, Y., Lu, B. Y., and Xu, J. K. (2015). Molecular design of DBT/DBF hybrid thiophenes $\pi$-conjugated systems and comparative study of their electropolymerization and optoelectronic properties: from comonomers to electrochromic polymers. Polym. Chem. 6, 4575-4587. doi: 10.1039/c5py00410a

Liu, M. N., Makuta, S., Tsuda, S., Russo, S., Seki, S., Terao, J., et al. (2017). Fluorene-thiophene copolymer wire on TiO2: mechanism achieving long charge separated state lifetimes. J. Phys. Chem. C 121:25672. doi: 10.1021/acs.jpcc.7b07295

Lu, B. Y., Jian, N. N., Qu, K., Hu, F. Q., Liu, X. M., Xu, J. X., et al. (2020). Stepwise enhancement on optoelectronic performances of polyselenophene via electropolymerization of mono-, Bi-, and triselenophene. Electrochim. Acta 340:135974. doi: 10.1016/j.electacta.2020.135974

Lu, B. Y., Zhen, S. J., Zhang, S. M., Xu, J. K., and Zhao, G. Q. (2014). Highly stable hybrid selenophene-3,4-ethylenedioxythiophene as electrically conducting and electrochromic polymers. Polym. Chem. 5, 4896-4908. doi: 10.1039/c4py00529e

Ming, S. L., Li, Z. Y., Zhen, S. J., Liu, P. P., Jiang, F. X., Nie, G. M., et al. (2020). High-performance D-A-D type electrochromic polymer with $\pi$ spacer applied in supercapacitor. Chem. Eng. J. 390:124572. doi: 10.1016/j.cej.2020. 124572

Sheberla, D., Patra, S., Wijsboom, Y. H., Sharma, S., Sheynin, Y., Haj-Yahia, A. E., et al. (2015). Conducting polyfurans by electropolymerization of oligofurans. Chem. Sci. 6, 360-371. doi: 10.1039/c4sc02664k

Sun, Y., Chien, S. C., Yip, H. L., Zhang, Y., Chen, K. S., Zeigler, D. F., et al. (2011). High-mobility low-bandgap conjugated copolymers based on indacenodithiophene and thiadiazolo[3,4-c]pyridine units for thin film transistor and photovoltaic applications. J. Mater. Chem. 21, 13247-13255. doi: $10.1039 / \mathrm{cljm} 11564 \mathrm{~b}$

Yang, K., He, T., D., Chen, X. Y., Cheng, S. Z. D., and Zhu, Y. (2014). Patternable conjugated polymers with latent hydrogen-bonding on the main chain. Macromolecules 47, 8479-8486. doi: 10.1021/ma501960t 
Yuan, Y., and Lei, A. W. (2020). Is electrosynthesis always green and advantageous compared to traditional methods? Nat. Commun. 11:802. doi: 10.1038/s41467-020-14322-z

Zhang, L., Colella, N. S., Liu, F., Trahan, S., Baral, J. K., Winter, H. H., et al. (2013). Synthesis, electronic structure, molecular packing/morphology evolution, and carrier mobilities of pure oligo-/poly(alkylthiophenes). J. Am. Chem. Soc. 135, 844-854. doi: 10.1021/ja3104796

Zhang, L., Luo, F. F., Li, W. J., Yan, S. M., Chen, Z. X., Zhao, R. Y., et al. (2019). Conjugation-broken thiophene-based electropolymerized polymers with well-defined structures: effect of conjugation lengths on electrochromic properties. Phys. Chem. Chem. Phys. 21, 24092-24100. doi: 10.1039/c9cp $04308 \mathrm{j}$

Zhou, N. J., Guo, X. G., Ortiz, R. P., Harschneck, T., Manley, E. F., Lou, S. J., et al. (2015). Marked consequences of systematic oligothiophene catenation in thieno[3,4-c]pyrrole-4,6-dione and bithiopheneimide photovoltaic copolymers. J. Am. Chem. Soc. 137, 12565-12579. doi: 10.1021/jacs.5b06462
Zhu, Y., Zhang, K., and Tieke, B. (2009). Electrochemical polymerization of Bis(3,4-ethylenedioxythiophene)-substituted 1,4-Diketo-3,6-diphenylpyrrolo[3,4-c]pyrrole (DPP) derivative. Macromol. Chem. Phys. 210, 431-439. doi: $10.1002 /$ macp. 200800507

Conflict of Interest: The authors declare that the research was conducted in the absence of any commercial or financial relationships that could be construed as a potential conflict of interest.

Copyright (c) $2020 \mathrm{Lin}, \mathrm{Li}$, Tao, Huang, Wu, Liu, Zhang, Wang and Liu. This is an open-access article distributed under the terms of the Creative Commons Attribution License (CC BY). The use, distribution or reproduction in other forums is permitted, provided the original author(s) and the copyright owner(s) are credited and that the original publication in this journal is cited, in accordance with accepted academic practice. No use, distribution or reproduction is permitted which does not comply with these terms. 